\section{Disseminated Mycobacterium avium complex in an adolescent with perinatally-acquired HIV infection}

\section{Nurul I. Hariadi, \\ R. Alexander Blackwood}

Division of Pediatric Infectious Diseases, University of Michigan, Ann Arbor, MI, USA

\begin{abstract}
Mycobacterium avium complex (MAC) is the most frequent nontuberculous mycobacteria implicated in opportunistic infections that define acquired immunodeficiency syndrome. With highly active antiretroviral therapy, disseminated MAC (dMAC) has become a rare entity. This unique case of dMAC was diagnosed in an adolescent with newly diagnosed perinatally-acquired HIV infection whose initial CD4 cell count was severely depleted and viral load was extremely high. While maximized treatment regimen had not been able to control his dMAC, improvement was noted when granulocyte macrophage colony-stimulating factor (GM-CSF) was added. GM-CSF should be considered as an adjunctive therapy in patients with refractory dMAC.
\end{abstract}

\section{Introduction}

Mycobacterium avium complex (MAC) is the most frequent nontuberculous mycobacteria (NTM) agent associated with acquired immunodeficiency syndrome (AIDS). ${ }^{1}$ The rate of disseminated MAC (dMAC) used to reach $16-40 \%$ in adult and $4.8 \%$ in pediatric AIDS patients during the early human immunodeficiency virus (HIV) pandemic..$^{2-4}$ However, in the era of highly active antiretroviral therapy (HAART), dMAC is an unusual entity. ${ }^{5}$ We present a unique case of dMAC in an adolescent with newly diagnosed perinatally-acquired HIV infection, with very low initial CD4 cell count and exceedingly high viral load. Virologic control was eventually reached after 10 months of HAART. However, the CD4 cell count has not fully recovered. The patient had also continued to have persistent dMAC despite maximized treatment regimen, which improved with addition of granulocyte macrophage colony-stimulating factor (GM-CSF).

\section{Case Report}

A thirteen-year old male was admitted following three months of dry cough, intermittent low-grade fever, and severe anemia. He had also stopped gaining weight and had been more fatigued for the past year. Past medical history was only pertinent for frequent upper respiratory infection since early childhood, with no previous serious illness, hospitalization, or surgery. There had been no recent travel or relatives visiting from abroad. He lived with maternal grandparents, as his mother had entered a drug rehabilitation program when he was five years old and reportedly died of hepatitis B complications. Physical exam was significant for low weight (5th percentile) and short stature (3rd percentile) for age, oral thrush, bilateral submandibular and supraclavicular lymphadenopathy, protuberant abdomen with splenomegaly $10 \mathrm{~cm}$ below the left costal margin, and decreased muscle mass. In addition, admission laboratory values were significant for lymphopenia (500 cells $/ \mathrm{mm}^{3}$ ) and anemia associated with reticulocytopenia (hemoglobin $3.9 \mathrm{~g} / \mathrm{dL}$, reticulocytes $2.8 \%$ ).

Evaluation of immune status showed positive HIV antibody by ELISA and Western Blot. Further inquiries confirmed maternal history of HIV infection and no behavioral risk factors for HIV in the patient. The initial viral load was $8,510,000$ HIV-1 RNA copies/mL plasma $\left(\log _{10}=6.93\right)$ and CD4 cell count was 3 cells/ $\mu \mathrm{L}(0.6 \%)$. Interferon gamma release assay was negative. The patient was started on HAART with lamivudine/zidovudine and lopinavir/ ritonavir; and aerosolized pentamidine for Pneumocystis jirovecii pneumonia (PCP) prophylaxis given marrow suppression risk with trimethoprim-sulfamethoxazole (TMP-SMX). MAC prophylaxis was initiated for severe immunocompromised state with clarithromycin. A bone marrow biopsy for severe anemia revealed normal cellularity and no evidence of malignancy; however, there were dense acid fast bacilli (AFB) occupying a significant portion of the marrow space (Figure 1). Isoniazid and ethambutol were added to clarithromycin prior to AFB speciation. Two weeks after HAART initiation, the viral load was still $>10,000,000 \quad$ copies $/ \mathrm{mL} \quad$ (estimated $85,000,000$ copies/mL following dilution) despite susceptibility to the regimen on viral genotyping.

Five weeks after diagnosis, the patient was readmitted with fever and diminished breath sound on right basal lung field. Bronchoscopy for concern of PCP revealed AFB that later was identified as MAC. He also developed painful swollen knees and
Correspondence: Nurul I. Hariadi, Banner Health Center Maricopa. 17900 N. Porter Rd. Maricopa, AZ 85138, USA.

Tel.: +1.520.233.2500 - Fax: +1.520.233.2574. E-mail: nurul.hariadi@gmail.com

Key words: HIV, disseminated Mycobacterium avium complex, adolescent, GM-CSF.

Acknowledgements: Lauren B. Smith, MD (Department of Pathology, University of Michigan) for histologic images and interpretations in the case report. Mark Vander Lugt, MD (Blood and Marrow Transplantation and Cellular Therapy, Children's Hospital of Pittsburgh) for expertise in HLH.

Contributions: Both NIH and RAB provided substantial contributions to the conception and revisions of the manuscript, and approved this final version for publication.

Conflict of interest: the authors declare no potential conflict of interest.

Received for publication: 11 September 2016. Revision received: 25 December 2016.

Accepted for publication: 27 December 2016

This work is licensed under a Creative Commons Attribution-NonCommercial 4.0 International License (CC BY-NC 4.0).

(C) Copyright N.I. Hariadi and R.A. Blackwood, 2017 Licensee PAGEPress, Italy

Infectious Disease Reports 2017; 9:6884

doi:10.4081/idr.2017.6884

ankles with synovial fluid cultures positive for MAC. Blood, urine, stool, and previous bone marrow cultures grew MAC as well. Seven weeks into treatment, he developed large right cervical lymphadenopathy and grossly bloody stools prompting a cervical lymph node biopsy and endoscopic biopsies that demonstrated MAC. Two months after diagnosis, his HIV load decreased to 4,900,000 copies/mL with CD4 cell count of 2 cells $/ \mathrm{mL}$. Serum drug levels confirmed therapeutic levels of his HAART. He was still transfusion dependent for anemia and the repeat bone marrow biopsy again revealed dense AFB. To optimize dMAC treatment, moxifloxacin and rifabutin were added to clarithromycin, and isoniazid was discontinued.

Four months into therapy, the patient developed fever, scleral icterus, neutropenia, elevated liver transaminases, and a ferritin level of $>16,500 \mathrm{ng} / \mathrm{mL}$ (above the test accuracy limit). Liver ultrasound showed diffuse gallbladder wall thickening and bone marrow biopsy revealed increased foamy histiocytes with occasional 
macrophages containing erythrocytes and cell debris, raising a concern for Hemophagocytic Lymphohistiocytosis (HLH). Although immune reconstitution inflammatory syndrome (IRIS) in conjunction with dMAC was also consistent with this progression, poor prognosis for untreated HLH in this clinical setting prompted the decision to initiate high dose dexamethasone followed by steroid taper. 6,7 Chemotherapy as a part of an HLH treatment protocol was held due to concerns related to his severe immunocompromized state, and amikacin was added to the dMAC regimen based on sensitivity of his MAC to clarithromycin and amikacin on susceptibility data.

The patient's viral load continued to decrease in response to HAART, to 258,000 RNA copies $/ \mathrm{mL}$ plasma $\left(\log _{10}=5.41\right)$ after four months, 2980 RNA copies $/ \mathrm{mL}$ $\left(\log _{10}=3.47\right)$ after six months. His CD4 cell count, however, never rose above 70 cells $/ \mathrm{mL}(13.3 \%)$. He also continued to be pancytopenic; requiring filgrastim [granulocyte-colony stimulating factor (G-CSF)], pRBC transfusions every 2-3 weeks despite the addition of erythropoietin, and platelet transfusion.

Seven months into therapy, the patient had also been diagnosed with invasive fun- gal rhinosinusitis with Aspergillus flavus, Alternaria sp., and Candida glabrata for which he received prolonged antifungals until his increased CD4 count was shown to be stable. In total, he received micafungin for nine months and liposomal amphotericin $\mathrm{B}$ for 13 months.

The patient's viral load eventually became undetected after 10 months of HAART, and his CD4 count increased to greater than 100 cells/mL after 15 months. Due to concern for iron overload from frequent transfusion, zidovudine was switched to stavudine to decrease marrow suppression at this point given evidences of adequate virologic and improving immunologic controls. Two months after the change, his anemia resolved and he has not required pRBC transfusion, although he still required G-CSF and platelet transfusion.

In regards to the patient's dMAC regimen, amikacin had to be discontinued due to marked hearing loss, while repeat bone marrow biopsies demonstrated persistence of extensive MAC. Based on potential benefit of sargramostim (GM-CSF) in refractory dMAC reported in case reports, ${ }^{8,9}$ G-CSF was changed to GM-CSF in month 16 from dMAC diagnosis. Bone marrow biopsy in month 18 showed normocellular bone marrow with negative AFB stain, although



Figure 1. Fite stain for acid fast bacilli on the bone marrow core biopsy (200x original magnification). The organisms, which are red, can be seen within histiocytes. there were still small granulomas (Figure 2). However, he had developed new cervical lymphadenopathy that was still positive for MAC.

Approximately 30 months after HIV diagnosis, the patient continues to have undetected viral load with CD4 count in 300 cells/mL range. He has not developed new lymphadenopathy and his AFB blood cultures remain negative although rifabutin had to be discontinued from his dMAC regimen due to bilateral uveitis. His platelet count has recovered and he has been able to maintain his WBC despite discontinuation of GM-CSF after 10 months.

\section{Discussion}

Most adolescents acquire HIV from sexual activity or intravenous drug use, with only $0.4 \%$ of adolescents and adults diagnosed with HIV had been infected via routes other than those related to sexual activity, including from perinatal exposure. ${ }^{10}$ There are very few case reports of perinatally-acquired HIV infection diagnosed in adolescence, ${ }^{11}$ which makes this case unique. While the initial presentation of our patient was classic for acquired immune deficiency, his immunosuppression level at the time of diagnosis was exceptionally severe. According to CDC classification, he met the AIDS-defining criteria with diagnosis of dMAC, CD4 cell count, and its percentage. ${ }^{12}$

Even though dMAC is not unexpected in our patient considering the level of his

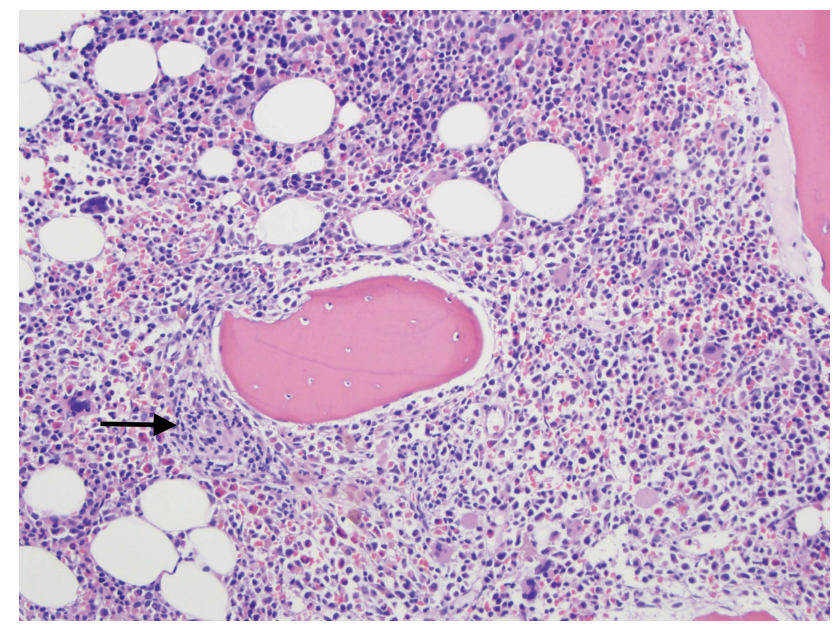

Figure 2. Hematoxylin and eosin section of the bone marrow after therapy, including GM-CSF (200x original magnification). The bone marrow shows normal cellularity with trilineage hematopoietic maturation and a small areas of granulomatous inflammation without necrosis (arrow). 
immunosuppression, it is a rare entity in HAART era. During early HIV pandemic, MAC was recognized to cause disseminated disease and death in advanced stages of immunosuppression with $\mathrm{CD}^{+}$counts fewer than 50 cells $/ \mu \mathrm{L}^{2-4}$ In the era of HAART, however, the most common clinical presentation of MAC infection in patients with good virological and immunological responses is IRIS, an inflammatory reaction that appears after initiating or changing HAART regimens which is temporally related to an increase in CD4 T-cell counts. ${ }^{13}$

IRIS is defined as a new onset or worsening symptoms of an infection or inflammatory condition after the start of HAART that cannot be explained by a newly acquired infection, predicted course of previously diagnosed infection, or adverse effects of drug therapy; with concordant viral load decrease $>1 \log _{10}$ copies/mL. ${ }^{14,15}$ The incidence of IRIS attributed to NTM was $3.5 \%$ among patients initiating HAART with a baseline $\mathrm{CD}^{+}$cell count of $<100$ cells $/ \mu \mathrm{L}$, mostly presenting as localized lymphadenitis or soft-tissue abscesses. ${ }^{16}$ Although IRIS is not commonly encountered in pediatric patients in the US thanks to early HIV diagnosis, it has been reported to occur in $21-38 \%$ of pediatric patients after initiation of HAART in other countries. ${ }^{17,18}$ Our patient's overwhelming dMAC initially raised the question of IRIS. However, his persistently high viral load did not fulfill the definition of IRIS, even though one might argue that it could have been secondary to the magnitude of its initial load on presentation. His CD4 cell count was not consistent with immune reconstitution either, which again could have also been attributed by the level of immune depletion at the time of diagnosis.

The patient's clinical course was complicated by severe anemia, neutropenia, and thrombocytopenia. Advanced HIV infection itself may cause anemia and neutropenia, ${ }^{19}$ and his bone marrow was significantly infiltrated by MAC. However, zidovudine has been associated with severe anemia and granulocytopenia, ${ }^{20}$ and with the risk of iron overload from frequent transfusion, the switch to a different nucleoside reverse transcriptase inhibitor with less marrow suppression was necessary.

Use of GM-CSF in dMAC has been described in limited case reports. ${ }^{8,9}$ In HIV patients, the host defect allowing MAC dissemination is specifically the failure of macrophages to kill phagocytized MAC, which is the theoretical base for use of GMCSF in dMAC. Although virologic control and immune reconstitution must have contributed to significant improvement in bone marrow findings from month eight to month 18 of dMAC treatment, we believe that the improvement might have also been attributable to addition of GM-CSF as an adjunctive treatment for dMAC.

\section{Conclusions}

dMAC in HIV patients is currently a rare entity in HAART era, and therefore the possibility of IRIS should also be assessed as a differential diagnosis. The use of GMCSF as an adjunctive treatment should be considered in patients with dMAC recalcitrant to more conservative approaches.

\section{References}

1. Horsburgh CR Jr., Selik RM. The epidemiology of disseminated nontuberculous mycobacterial infection in the acquired immunodeficiency syndrome (AIDS). Am Rev Respir Dis 1989; 139:4-7.

2. Havlik JA Jr., Horsburgh CR Jr., Metchock B, et al. Disseminated Mycobacterium avium complex infection: clinical identification and epidemiologic trends. J Infect Dis 1992;165: 577-80.

3. Horsburgh CR Jr., Caldwell MB, Simonds RJ. Epidemiology of disseminated nontuberculous mycobacterial disease in children with acquired immunodeficiency syndrome. Pediatr Infect Dis J 1993;12:219-22.

4. Nightingale SD, Byrd LT, Southern PM, et al. Incidence of Mycobacterium avium-intracellulare complex bacteremia in human immunodeficiency virus-positive patients. $\mathrm{J}$ Infect Dis 1992;165:1082-5.

5. Gona P, Van Dyke RB, Williams PL, et al. Incidence of opportunistic and other infections in HIV-infected children in the HAART era. JAMA. 2006;296:292300.

6. Grateau G, Bachmeyer C, Blanche P, et al. Haemophagocytic syndrome in patients infected with the human immunodeficiency virus: nine cases and a review. J Infect 1997;34:219-25.

7. Henter JI, Horne A, Arico M, et al. HLH-2004: Diagnostic and therapeutic guidelines for hemophagocytic lymphohistiocytosis. Pediatr Blood Cancer 2007;48:124-31.

8. Nannini EC, Keating M, Binstock P, et al. Successful treatment of refractory disseminated Mycobacterium avium complex infection with the addition of linezolid and mefloquine. J Infect 2002; 44:201-3.

9. de Silva TI, Cope A, Goepel J, Greig JM. The use of adjuvant granulocytemacrophage colony-stimulating factor in HIV-related disseminated atypical mycobacterial infection. J Infect 2007; 54:e207-10.

10. Centers for Disease Control and Prevention. HIV surveillance report. 2008. Available from: https://www. cdc.gov/hiv/pdf/library/reports/surveillance/cdc-hiv-surveillance-report-2008vol-20.pdf. Accessed: January 2017.

11. Barroso H, Araujo F, Gomes MH, et al. Phylogenetic demonstration of two cases of perinatal human immunodeficiency virus type 2 infection diagnosed in adulthood. AIDS Res Hum Retroviruses 2004;20:1373-6.

12. Schneider E, Whitmore S, Glynn KM, et al. Revised surveillance case definitions for HIV infection among adults, adolescents, and children aged $<18$ months and for HIV infection and AIDS among children aged 18 months to $<13$ years: United States, 2008. MMWR Recomm Rep 2008;57:1-12.

13. Corti M, Palmero D. Mycobacterium avium complex infection in HIV/AIDS patients. Expert Rev Anti Infect Ther 2008;6:351-63.

14. Robertson J, Meier M, Wall J, et al. Immune reconstitution syndrome in HIV: validating a case definition and identifying clinical predictors in persons initiating antiretroviral therapy. Clin Infect Dis 2006;42:1639-46.

15. Haddow LJ, Easterbrook PJ, Mosam A, et al. Defining immune reconstitution inflammatory syndrome: evaluation of expert opinion versus 2 case definitions in a South African cohort. Clin Infect Dis 2009;49:1424-32.

16. Phillips P, Bonner S, Gataric N, et al. Nontuberculous mycobacterial immune reconstitution syndrome in HIV-infected patients: spectrum of disease and long-term follow-up. Clin Infect Dis 2005;41:1483-97.

17. Smith K, Kuhn L, Coovadia A, et al. Immune reconstitution inflammatory syndrome among HIV-infected South African infants initiating antiretroviral therapy. AIDS 2009;23:1097-107.

18. Orikiiriza J, Bakeera-Kitaka S, Musiime V, et al. The clinical pattern, prevalence, and factors associated with immune reconstitution inflammatory syndrome in Ugandan children. AIDS 2010;24:2009-17.

19. Sterling TR, Chaisson RE. General clinical manifestations of human immunodeficiency virus infection (including the 
acute retroviral syndrome and oral, cutaneous, renal, ocular, metabolic, and cardiac diseases). In: Mandell GL, Bennett JE, Dolin R, eds. Mandell: Mandell, Douglas, and Bennett's Principles and Practice of Infectious
Diseases. 1st ed. London: Churchill Livingstone; 2009. pp 1705-26.

20. Tsibris AMN, Hirsch MS. Antiretroviral therapy for human immunodeficiency virus infection. In: Mandell GL, Bennett JE, Dolin R, editor. Mandell:
Mandell, Douglas, and Bennett's Principles and Practice of Infectious Diseases. 1st ed. London: Churchill Livingstone; 2009. pp 1833-54. 